CNS Spectrums (2019), 24, 249-257. C Cambridge University Press 2017. The online version of this article is published within an Open Access environment subject to the conditions of the Creative Commons Attribution-NonCommercial-ShareAlike licence $<$ http://creative commons.org/licenses/by-nc-sa/4.0/>. The written permission of Cambridge University Press must be obtained for commercial re-use. doi:10.1017/S1092852917000761

\title{
Efficacy of vortioxetine in working patients with generalized anxiety disorder
}

\author{
Michael Cronquist Christensen, ${ }^{1}$ Henrik Loft, ${ }^{1}$ Ioana Florea,${ }^{\prime}$ and \\ Roger S. McIntyre ${ }^{2 *}$
}

\footnotetext{
${ }^{1}$ H. Lundbeck A/S, Valby, Denmark

${ }^{2}$ Mood Disorders Psychopharmacology Unit, University Health Network, University of Toronto, Toronto, Ontario, Canada
}

Background. Vortioxetine is an approved antidepressant that has also demonstrated positive effects on anxiety symptoms in subjects with generalized anxiety disorder (GAD). This post-hoc analysis evaluates the efficacy of vortioxetine in GAD subjects who are working and/or pursuing an education.

Methods. In study NCT00744627, 301 GAD subjects were randomized to vortioxetine $5 \mathrm{mg}$ or placebo for 8 weeks. Efficacy measures included the Hamilton Anxiety Rating Scale (HAM-A) total score, response/remission, global functioning (Sheehan Disability Scale [SDS]), and quality of life (Short Form-36 Health Survey). In study NCT00788034, 687 GAD subjects were treated open-label with vortioxetine 5 or $10 \mathrm{mg}$ for 20 weeks, after which subjects in remission were randomized to fixed-dose of vortioxetine $(5 \mathrm{or} 10 \mathrm{mg}$ ) or placebo for at least 24 weeks. The primary endpoint was time to relapse. Analyses were completed in subjects working and/or pursuing an education at study entry and the full analysis set.

Results. In study NCT00744627, the effect of vortioxetine versus placebo on HAM-A total score was $-4.3(p=0.0005)$ in working subjects $(60 \%$ of total), while the effect in the total population was $-3.8(p=0.0001)$. The effect was greatest in subjects in professional $(-4.5, p=0.0130)$ and associate professional positions $(-7.6, p=0.0086)$. Greater effects in terms of response, remission, and the SDS and SF-36 were also observed. In NCT00788034, working subjects $(69 \%$ of total) randomized to placebo were significantly more likely to relapse than subjects treated with vortioxetine (hazard ratio $=2.9 ; p<0.001)$, while the hazard ratio in the total population was $2.7(p<0.0001)$.

Conclusions. The beneficial effects of vortioxetine on anxiety symptoms, functioning, and quality of life are greater in adults with GAD who are working and/or pursuing an education versus the full GAD study population.

Received 10 April 2017; Accepted 7 September 2017; First published online 30 October 2017

Key words: Vortioxetine, working population, generalized anxiety disorder, functioning, quality of life, pharmacological treatment.

\section{Introduction}

Generalized anxiety disorder $(\mathrm{GAD})$ is prevalent (12-month and lifetime prevalence of 3.1 and $5.1 \%$, respectively) and associated with significant impairments in psychosocial functioning, including work functioning. ${ }^{1,2}$ Many individuals with GAD have a comorbid psychiatric disorder, with major depressive disorder (MDD) being the most common (current,

* Address for correspondence: Roger S. McIntyre, Department of Psychiatry and Pharmacology, Mood Disorders Psychopharmacology Unit, University of Toronto, University Health Network, 399 Bathurst Street, Toronto, Ontario, M5T 2S8, Canada.

(Email: roger.mcintyre@uhn.ca) 12-month, or lifetime). ${ }^{3}$ The magnitude of psychosocial impairment in individuals with GAD without comorbidity is similar to that of individuals with MDD; thus, $17-19 \%$ of individuals with GAD have been reported as being disabled 3 or more days per month, and the percentage is substantially greater among individuals with GAD and comorbid MDD (approximately 34\%). ${ }^{1}$ Workplace impairment studies have reported a reduction in overall work productivity in the range of $11-49 \%$ in more than $23 \%$ of individuals with pure GAD and more than $50 \%$ in $20 \%$ of individuals with comorbid MDD. ${ }^{4}$ Since many individuals with GAD are working, this implies substantial costs to employers and society at large. 
A number of theoretical frameworks, such as the demand/control/support (DCS) model and the effort/ reward imbalance (ERI) model help us understand vulnerability to anxiety in the workplace. ${ }^{5,6}$ The DCS model, for instance, hypothesizes that "job strain" occurs when high physical and psychological demands combine with low levels of control of the daily work. ${ }^{7}$ Control in this context more specifically implies the ability to make decisions (decision authority) and use personal skills (skill discretion) in executing the job. Such control is argued to be a resource that facilitates both health and productivity. ${ }^{8}$ For anxiety specifically, one can speculate that individuals who are higher in the organizational chart of the company presumably have a stronger internal locus of control serving as resiliency for anxiety. The longitudinal Whitehall II study, for instance, found a significant association between high levels of perceived social support at work, control at work, job variety, and skills use with less psychiatric morbidity. ${ }^{9}$ A similar effect was observed in this study when investigating the impact of job security, including chronic perceived job insecurity. ${ }^{10}$

Vortioxetine is an approved antidepressant with a multimodal mechanism of action that has also demonstrated positive effects on anxiety symptoms in patients with GAD in both the short and long term. ${ }^{11,12}$ Overall, the investigation of vortioxetine in GAD comprised a total of five pivotal trials, including three short-term US studies that did not meet their primary endpoint. ${ }^{13}$ We have previously demonstrated a more pronounced effect of vortioxetine in the working MDD population, both on depressive symptoms and cognitive functioning, with the greatest effect observed among individuals in "professional"-type positions (e.g., managers). ${ }^{14}$ A non-mutually-exclusive explanatory framework proposed for these findings is that working patients in such positions have relatively higher levels of resiliency, motivation, and/or internal locus of control, and thus have a greater potential for cognitive improvement during treatment with vortioxetine.

Given the substantial overlap between MDD and GAD, we hypothesized for this post-hoc analysis that vortioxetine's effects on anxiety symptoms, global functioning, and quality of life may also be more pronounced in GAD patients who are working compared to the effect already reported in a full adult GAD population. Additionally, given that individuals who are experiencing higher internal locus of control may be less prone to anxiety and possibly more likely to benefit from anxiolytic interventions, we also hypothesized a differential therapeutic benefit among the different subgroups of working patients according to type of employment. As this study is post hoc in its statistical approach, we decided to delimit inclusion of studies that met their a-priori endpoint. The rationale for this approach is that we wanted to include only those studies that demonstrated sufficient assay sensitivity, providing the opportunity to evaluate the posthoc questions posed herein.

\section{Materials and Methods}

\section{Acute GAD study}

The full details of study NCT00744627 are provided in Bidzan et al. (2012). ${ }^{11}$ In short, this was a phase 3, multinational, randomized, placebo-controlled, fixeddose, 8-week study in 301 outpatients, of either sex, aged 18-65 years with a primary diagnosis of GAD according to DSM-IV-TR criteria, as well as a Hamilton Anxiety Scale (HAM-A) total score $\geq 20$ at screening and baseline, HAM-A scores $\geq 2$ on both items 1 (anxious mood) and 2 (tension) at screening and baseline, and a MontgomeryÅsberg Depression Rating Scale (MADRS) total score $\leq 16$ at screening and baseline. Eligible subjects were randomized $(1: 1)$ to receive $5 \mathrm{mg}$ of vortioxetine or placebo, once daily, during the 8-week double-blind treatment period. Individuals were excluded if they had any current psychiatric disorder other than GAD. The study was conducted at 47 sites in Estonia, Germany, Latvia, Lithuania, Poland, Romania, Russia, Ukraine, and South Africa between September of 2008 and July of 2009. Working status and type of employment were assessed at baseline according to the Health Economic Assessment (HEA) questionnaire. ${ }^{15}$ The HEA records working status (full-time work/school; part-time work/school; unemployed; nonworking spouse; retired; or other) as well as type of job, if working (manager/administrator, professional [e.g., health, teaching, legal], associate professional [e.g., technical, nursing], clerical worker/secretary, skilled laborer [e.g., building, electrical/factory worker], services/sales [e.g., retail], and other). In this analysis, the working population included full- and part-time workers, or those in school. In terms of analysis by type of employment, we combined the employment categories in the following manner. Manager/administrator and professional were grouped into a category termed "professional"; associate professional and clerical worker/ secretary were all termed "associate professionals"; services/sales and other were all termed "skilled laborers." All analyses were performed in the full study population (full analysis set [FAS]), the working population, and the respective subgroups of working patients.

\section{GAD relapse prevention study}

The full details of study NCT00788034 are provided in Baldwin et al. (2012). ${ }^{12}$ In short, this was a double-blind, randomized, placebo-controlled relapse prevention study in 687 outpatients, of either sex, aged 18 to 75 years, with a primary diagnosis of GAD according to DSMIV-TR criteria. Similar to the acute GAD study, only patients with a HAM-A total score of at least 20, a HAMA score of at least 2 on items 1 and 2, and an MADRS total score $\leq 16$ at both the screening and baseline visits 
were included. Patients were excluded if they had any current psychiatric disorder other than GAD. The patient's working status was also measured by the HEA, as in the acute GAD study. This study included two consecutive periods: a 20-week open-label, flexible-dose treatment period with vortioxetine 5 or $10 \mathrm{mg}$, and a double-blind, fixed-dose, placebo-controlled treatment period of 24-56 weeks with vortioxetine 5 or $10 \mathrm{mg}$ or placebo. The initial dose of vortioxetine was $5 \mathrm{mg} /$ day. During the open-label period (from weeks 2 to 8 ), the investigator could, if clinically indicated, increase the dose to $10 \mathrm{mg}$ /day once and could decrease it again to $5 \mathrm{mg} /$ day in connection with a study visit. From week 8 and onward, the dose remained fixed. Patients who had responded to treatment (HAM-A total score $\leq 10$ at both weeks 16 and 20) were randomized to the double-blind, placebo-controlled, fixed-dose treatment period. Patients were randomized equally $(1: 1)$ to vortioxetine or placebo in the double-blind period. Patients randomized to vortioxetine continued on the final dose ( $5 \mathrm{or} 10 \mathrm{mg} /$ day) that was fixed from week 8 in the open-label period, whereas patients randomized to placebo were switched abruptly to placebo.

Throughout the double-blind period, investigators evaluated the occurrence of relapse, defined either as a HAM-A total score of at least 15 or an insufficient therapeutic response, according to their clinical judgment. As all patients were to complete the study simultaneously, non-withdrawn patients continued in the study until the last patient completed 24 weeks of double-blind treatment. The maximum duration of the double-blind period was 56 weeks. The study was conducted at 80 centers in Argentina, Chile, Colombia, Costa Rica, Estonia, Finland, France, Hungary, Russia, and South Africa between October of 2008 and June of 2010.

Both studies were conducted in accordance with the International Conference on Harmonisation principles of Good Clinical Practice (1996) and the Declaration of Helsinki (2002). The local ethics committees approved the trial designs, and eligible patients provided written informed consent before participating.

\section{Clinical assessments}

In the acute GAD study, efficacy endpoints included the Hamilton Anxiety Rating Scale (HAM-A), the Clinical Global Impression-Severity Scale (CGI-S), the Clinical Global Impression-Improvement Scale (CGI-I), the patient-reported Hospital Anxiety and Depression Scale (HAD), the Short Form-36 Health Survey (SF-36), and the Sheehan Disability Scale (SDS). Data was collected at week 1, 2, 4 and 8 after randomization. In the $\mathrm{GAD}$ relapse prevention study, efficacy endpoints included the HAM-A, the SF-36, and the SDS. Data were collected in the doubleblind period at 1,2 , and 4 weeks after randomization (baseline II) and then at 4-week intervals.

\section{Statistical analysis}

In the acute GAD study, the primary endpoint (change from baseline HAM-A total score to week 8) was analyzed by comparing vortioxetine $5 \mathrm{mg}$ and placebo in the FAS at all assessment points using a mixed model for repeated measurements (MMRM; observed cases [OC]). Items 1 (anxious mood) and 2 (tension) were also analyzed separately. Change from baseline in the CGI-S, HAD, SDS, and SF-36 were analyzed as continuous variables similar to the primary endpoint. The relevant baseline value was used as the covariate adjustment in the analyses. A HAM-A response (decrease of $\geq 50 \%$ from baseline in HAM-A total score) and remission (HAM-A total score of 57 ) were analyzed by logistic regression, adjusting for baseline score and treatment using last observation carried forward (LOCF) data. CGI-S remission (CGI-S severity $\leq 2$ ) and CGI-I improvement (CGI-I $\leq 2$ ) were also analyzed by logistic regression, adjusting for baseline score and treatment using LOCF data. In the relapse prevention study, the primary efficacy variable was the time to relapse during the double-blind period. The primary efficacy analysis employed a Cox model using an exact method to handle ties on the basis of the FAS. Withdrawals due to reasons other than lack of efficacy (relapse) were considered as non-relapsed, and the date of withdrawal was considered as the censoring time. HAM-A, SF-36, and SDS scores were evaluated in the double-blind period by considering change from time of randomization (baseline II) up to week 48. All endpoints were analyzed at all timepoints by logistic regression, adjusting for baseline score and treatment using LOCF.

\section{Results}

\section{Baseline characteristics}

In the acute $\mathrm{GAD}$ study, $60 \%(n=180)$ of the 301 subjects indicated that they were working or pursuing an education (full- or part-time) at baseline. In total 71 subjects $(39 \%)$ were in the professional group, $38(21 \%)$ in the associate professional group, and 65 $(36 \%)$ in the skilled laborer group. In the relapse prevention study, 69\% $(n=481)$ of the 687 subjects reported that they were working or pursuing an education at baseline, with 154 subjects $(32 \%)$ in the professional group, $95(20 \%)$ in the associate professional group, and $191(40 \%)$ in the skilled laborer group (Table 1). There were no substantial differences between groups in baseline demographic or clinical characteristics, nor between the working population overall and the total study population in any of the two studies.

\section{Clinical outcomes}

In the acute $\mathrm{GAD}$ study, the effect of vortioxetine versus placebo in change from baseline in HAM-A total score to 
TABLE 1. Demographics and baseline clinical characteristics (FAS) for the acute GAD study (Bidzan et al., 2012 ${ }^{11}$ ) and the relapse prevention study (Baldwin et al., 2012 ${ }^{12}$ ) (FAS, mean scores)

\begin{tabular}{|c|c|c|c|c|c|c|c|c|c|c|}
\hline & \multicolumn{2}{|c|}{ All individuals } & \multicolumn{2}{|c|}{ Working patients } & \multicolumn{2}{|c|}{ Professionals } & \multicolumn{2}{|c|}{ Associate professionals } & \multicolumn{2}{|c|}{ Skilled laborers } \\
\hline & Mean $\pm S D$ & $n$ & Mean $\pm S D$ & $n$ & Mean $\pm S D$ & $n$ & Mean $\pm S D$ & $n$ & Mean $\pm S D$ & $n$ \\
\hline \multicolumn{11}{|l|}{ Acute GAD study } \\
\hline Mean age $\pm S D$, years & $45.1 \pm 13.8$ & 301 & $41.3 \pm 11.3$ & $180(60.2 \%)$ & $41.1 \pm 11.3$ & 71 & $43.1 \pm 10.5$ & 38 & $42.2 \pm 10.7$ & 65 \\
\hline Gender, \% female & 65.1 & 196 & 61.1 & 110 & 63.4 & 71 & 86.8 & 33 & 43.1 & 28 \\
\hline HAM-A total & $26.6 \pm 3.9$ & 301 & $26.5 \pm 4.0$ & 180 & $25.8 \pm 3.4$ & 71 & $27.0 \pm 4.0$ & 38 & $26.9 \pm 4.7$ & 65 \\
\hline MADRS total & $12.2 \pm 2.3$ & 301 & $12.1 \pm 2.3$ & 180 & $11.8 \pm 2.5$ & 71 & $12.1 \pm 2.4$ & 38 & $12.4 \pm 2.2$ & 65 \\
\hline SDS total & $17.2 \pm 6.0$ & 248 & $16.9 \pm 5.7$ & 176 & $16.4 \pm 5.6$ & 70 & $15.8 \pm 6.4$ & 36 & $18.0 \pm 5.0$ & 64 \\
\hline SF-36 total & $32.8 \pm 9.9$ & 301 & $33.3 \pm 10.0$ & 180 & $35.5 \pm 9.9$ & 71 & $31.9 \pm 11.0$ & 38 & $31.8 \pm 9.0$ & 65 \\
\hline HAD-A & $14.1 \pm 3.0$ & 301 & $13.9 \pm 3.1$ & 180 & $13.6 \pm 3.2$ & 71 & $13.3 \pm 3.0$ & 38 & $14.6 \pm 2.8$ & 65 \\
\hline$H A D-D$ & $7.6 \pm 4.0$ & 301 & $7.1 \pm 3.9$ & 180 & $6.9 \pm 4.0$ & 71 & $7.5 \pm 3.6$ & 38 & $7.1 \pm 4.0$ & 65 \\
\hline CGI-S & $4.5 \pm 0.7$ & 301 & $4.4 \pm 0.7$ & 180 & $4.4 \pm 0.6$ & 71 & $4.5 \pm 0.7$ & 38 & $4.5 \pm 0.7$ & 65 \\
\hline \multicolumn{11}{|c|}{ GAD relapse prevention study } \\
\hline Mean age $\pm S D$, years & $43.3 \pm 13.3$ & 693 & $40.3 \pm 11.5$ & $481(69.4 \%)$ & $43.4 \pm 10.2$ & 154 & $42.2 \pm 10.7$ & 95 & $40.0 \pm 11.1$ & 191 \\
\hline Gender, \% female & 63.1 & 437 & 58.6 & 282 & 51.9 & 80 & 73.7 & 70 & 54.5 & 104 \\
\hline HAM-A total I & $28.4 \pm 4.6$ & 693 & $28.0 \pm 4.3$ & 481 & $27.4 \pm 3.7$ & 154 & $28.0 \pm 4.5$ & 95 & $28.7 \pm 4.6$ & 191 \\
\hline HAM-A total II & $4.5 \pm 2.9$ & 459 & $4.4 \pm 2.9$ & 319 & $3.9 \pm 2.8$ & 101 & $4.5 \pm 3.0$ & 64 & $4.7 \pm 2.7$ & 131 \\
\hline SDS total I & $16.6 \pm 6.8$ & 610 & $16.5 \pm 6.5$ & 471 & $16.0 \pm 6.0$ & 150 & $16.0 \pm 6.6$ & 92 & $17.4 \pm 6.8$ & 188 \\
\hline SDS total II & $4.9 \pm 5.9$ & 440 & $4.4 \pm 5.2$ & 318 & $4.4 \pm 4.5$ & 101 & $3.9 \pm 4.5$ & 63 & $4.9 \pm 6.2$ & 131 \\
\hline HAD-A I & $13.1 \pm 3.7$ & 690 & $13.0 \pm 3.7$ & 478 & $12.6 \pm 3.5$ & 154 & $12.9 \pm 3.7$ & 95 & $13.5 \pm 3.7$ & 188 \\
\hline HAD-A II & $5.1 \pm 3.5$ & 459 & $5.0 \pm 3.5$ & 319 & $5.0 \pm 3.1$ & 101 & $5.0 \pm 3.4$ & 64 & $5.1 \pm 3.8$ & 131 \\
\hline HAD-D I & $8.3 \pm 4.1$ & 690 & $8.0 \pm 4.0$ & 478 & $8.1 \pm 3.7$ & 154 & $7.8 \pm 4.0$ & 95 & $8.5 \pm 4.3$ & 188 \\
\hline HAD-D II & $3.4 \pm 3.6$ & 459 & $3.2 \pm 3.4$ & 319 & $3.1 \pm 3.0$ & 101 & $3.3 \pm 3.1$ & 64 & $3.5 \pm 3.9$ & 131 \\
\hline CGI-S I & $4.7 \pm 0.7$ & 693 & $4.6 \pm 0.7$ & 481 & $4.6 \pm 0.6$ & 154 & $4.6 \pm 0.7$ & 95 & $4.6 \pm 0.7$ & 191 \\
\hline CGI-S II & $1.6 \pm 0.7$ & 459 & $1.6 \pm 0.7$ & 319 & $1.6 \pm 0.7$ & 101 & $1.6 \pm 0.8$ & 64 & $1.6 \pm 0.7$ & 131 \\
\hline
\end{tabular}

CGI-S = Clinical Global Impression-Severity; HAD-A=Hospital Anxiety and Depression Scale (HAD) Anxiety subscore; HAD-D = Hospital Anxiety and Depression Scale (HAD) Depression subscore; HAM-A = Hamilton Anxiety Rating Scale; I = first baseline score at study entry; II = baseline score after 20 weeks of treatment and prior to randomization; MADRS = Montgomery-Åsberg Depression Rating Scale; SDS = Sheehan Disability Scale; SF36; Short Form (36) Health Survey.

week 8 was $-4.3(p=0.0005)$ in working patients, while the difference versus placebo in the total population was -3.8 $(p=0.0001)$ (Table 2, Figure 1). For professionals, associate professionals, and skilled laborers, the effect of vortioxetine on HAM-A total score versus placebo was $-4.5(p=0.0130),-7.6 \quad(p=0.0086)$, and -1.8 $(p=0.4547)$, respectively. For both HAM-A items 1 (anxious mood) and 2 (tension), a significant effect was also observed in the working $\mathrm{GAD}$ population overall and among professionals and associate professionals. For response based on the HAM-A $(\geq 50 \%$ reduction in HAM-A total score from baseline), a significant effect was also observed in the working population and among professionals (Table 3). In terms of response defined using the broader global assessment of clinical improvement over 8 weeks of treatment (CGI-I improvement $\leq 2$ ), a significant effect was observed in working patients overall (odds ratio $[O R]=2.2, p=0.0088$ ) and among associate professionals $(O R=6.1, p=0.0120)$. The effect in the total population had an $O R=2.5$ $(p=0.0002)$. For remission based on global assessment of clinical impression (CGI-S severity $\leq 2$ ), a significant effect was also observed in working patients overall
$(O R=2.1, p=0.0260)$ and among associate professionals $(O R=11.9, p=0.0071)$, while the effect in the total population had an $O R=1.9(p=0.0169)$.

In regard to patient-reported outcomes, the effect of vortioxetine versus placebo on the HADS anxiety subscore was $-1.8(p=0.0127)$ in working patients overall and $-2.7(p=0.0206)$ among professionals and $-2.9(p=0.0531)$ among associate professionals (Table 2). The effect in the total population was -2.4 $(p<0.0001)$. A similar pattern of results was observed for the SF-36 for all three domains as well as the SDS, in particular among professionals. The effect on SDS total score was $-4.0(p=0.0199)$ and $-1.7(p=0.0041)$ for the SDS family subscore, while the effect in the total study population was $-2.0(p=0.0307)$ and $-1.0(p=0.0006)$, respectively.

In the relapse prevention study, working GAD patients randomized to placebo were significantly more likely to relapse than those randomized to vortioxetine with a hazard ratio of 2.9 (95\% confidence interval [95\% CI]: $1.7-4.7, p<0.0001)$. The hazard ratio for the total study population was 2.7 (95\% CI: $1.8-4.1$, $p<0.0001)$. Among professionals, associate professionals, 


\begin{tabular}{|c|c|c|c|c|c|c|c|c|c|c|}
\hline \multirow[t]{2}{*}{ Outcome measure } & \multicolumn{2}{|c|}{$\begin{array}{l}\text { All individuals } \\
\quad(n=301)\end{array}$} & \multicolumn{2}{|c|}{$\begin{array}{l}\text { Working all } \\
(n=180)\end{array}$} & \multicolumn{2}{|c|}{$\begin{array}{l}\text { Professionals } \\
\qquad(n=71)\end{array}$} & \multicolumn{2}{|c|}{$\begin{array}{l}\text { Associate professionals } \\
\qquad(n=38)\end{array}$} & \multicolumn{2}{|c|}{$\begin{array}{l}\text { Skilled laborers } \\
\qquad(n=65)\end{array}$} \\
\hline & Mean $\pm S E$ & Value of $p$ & Mean $\pm S E$ & Value of $p$ & Mean $\pm S E$ & Value of $p$ & Mean $\pm S E$ & Value of $p$ & Mean $\pm S E$ & Value of $p$ \\
\hline \multicolumn{11}{|l|}{ Acute GAD study } \\
\hline HAM-A total & $-3.8 \pm 1.0$ & 0.0001 & $-4.3 \pm 1.2$ & 0.0005 & $-4.5 \pm 1.7$ & 0.0130 & $-7.6 \pm 2.7$ & 0.0086 & $-1.8 \pm 2.4$ & 0.4547 \\
\hline HAM-A item 1 & $-0.4 \pm 0.1$ & 0.0004 & $-0.5 \pm 0.1$ & 0.0006 & $-0.5 \pm 0.2$ & 0.0197 & $-1.1 \pm 0.3$ & 0.0012 & $-0.2 \pm 0.3$ & 0.4289 \\
\hline HAM-A item 2 & $-0.4 \pm 0.1$ & 0.0002 & $-0.4 \pm 0.1$ & 0.0008 & $-0.6 \pm 0.2$ & 0.0033 & $-1.0 \pm 0.3$ & 0.0013 & $-0.1 \pm 0.2$ & 0.8104 \\
\hline SDS total & $-2.0 \pm 0.9$ & 0.0307 & $-1.9 \pm 1.1$ & 0.0734 & $-4.0 \pm 1.7$ & 0.0199 & $-3.4 \pm 2.6$ & 0.1927 & $1.6 \pm 1.9$ & 0.3827 \\
\hline SDS work & $-0.4 \pm 0.3$ & 0.1721 & $-0.5 \pm 0.4$ & 0.1583 & $-1.0 \pm 0.6$ & 0.0903 & $-0.5 \pm 0.8$ & 0.5266 & $0.5 \pm 0.7$ & 0.5135 \\
\hline SDS family & $-1.0 \pm 0.3$ & 0.0006 & $-0.9 \pm 0.4$ & 0.0225 & $-1.7 \pm 0.6$ & 0.0041 & $-1.6 \pm 0.9$ & 0.0950 & $0.6 \pm 0.7$ & 0.4190 \\
\hline SDS social & $-0.8 \pm 0.3$ & 0.0066 & $-0.6 \pm 0.4$ & 0.1263 & $-1.1 \pm 0.6$ & 0.0754 & $-1.1 \pm 0.9$ & 0.2119 & $0.6 \pm 0.7$ & 0.4296 \\
\hline SF-36 mental health index & $4.6 \pm 1.3$ & 0.0006 & $4.3 \pm 1.6$ & 0.0073 & $6.0 \pm 2.5$ & 0.0202 & $7.8 \pm 4.2$ & 0.0721 & $0.7 \pm 2.9$ & 0.7988 \\
\hline SF-36 role-emotional & $4.2 \pm 1.4$ & 0.0024 & $3.6 \pm 1.7$ & 0.0373 & $4.6 \pm 3.0$ & 0.1390 & $6.9 \pm 4.5$ & 0.1374 & $0.1 \pm 2.9$ & 0.9605 \\
\hline SF-36 social functioning & $3.8 \pm 1.2$ & 0.0017 & $3.0 \pm 1.5$ & 0.0500 & $4.5 \pm 2.2$ & 0.0524 & $1.1 \pm 3.6$ & 0.7549 & $2.3 \pm 3.2$ & 0.4802 \\
\hline HAD-A & $-2.4 \pm 0.6$ & $<0.0001$ & $-1.8 \pm 0.7$ & 0.0127 & $-2.7 \pm 1.1$ & 0.0206 & $-2.9 \pm 1.5$ & 0.0531 & $0.4 \pm 1.5$ & 0.7862 \\
\hline HAD-D & $-1.7 \pm 0.4$ & $<0.0001$ & $-1.5 \pm 0.5$ & 0.0016 & $-0.9 \pm 0.8$ & 0.2727 & $-1.9 \pm 1.2$ & 0.1068 & $-1.1 \pm 0.8$ & 0.1641 \\
\hline CGI-S & $-0.5 \pm 0.1$ & 0.0002 & $-0.6 \pm 0.2$ & 0.0007 & $-0.5 \pm 0.3$ & 0.0717 & $-1.3 \pm 0.4$ & 0.0012 & $-0.4 \pm 0.3$ & 0.2613 \\
\hline
\end{tabular}

CGI-S = Clinical Global Impression-Severity; FAS = full analysis set; HAD-A=Hospital Anxiety and Depression Scale Anxiety subscore; HAD-D = Hospital Anxiety and Depression Scale Depression subscore; HAM-A = Hamilton Anxiety Rating Scale; MMRM= mixed model for repeated measurements; $0 \mathrm{C}=0 \mathrm{bserved}$ cases; $\mathrm{SDS}=\mathrm{Sheehan}$ Disability Scale; SF-36 = Short Form (36) Health Survey.

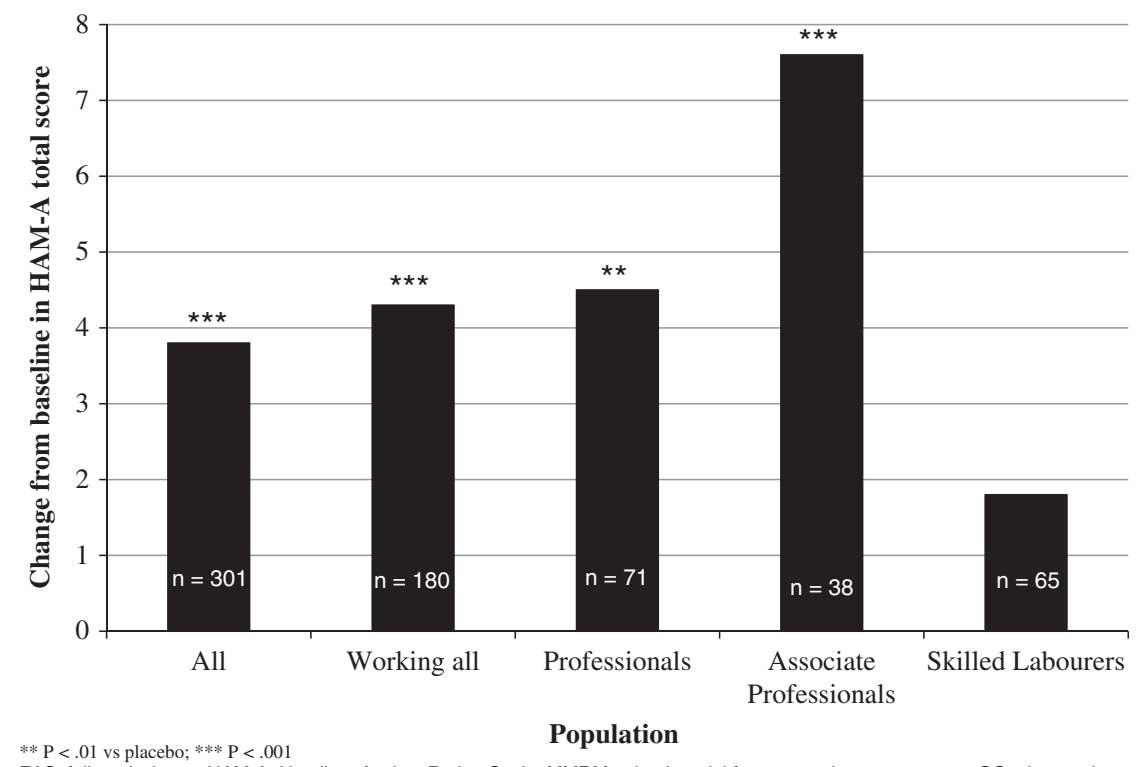

FAS; full analysis set. HAM-A; Hamilton Anxiety Rating Scale. MMRM; mixed model for repeated measurements. OC; observed cases.

FIGURE 1. Change from baseline in HAM-A total score to week 8, vortioxetine vs. placebo (MMRM, OC, FAS), total study population, working population, and by type of employment (Bidzan et al., 2012 ${ }^{11}$ ).

and skilled laborers, the hazard ratio was $2.5(95 \% \mathrm{CI}$ : $1.2-5.3, p=0.0199), 17.7$ (95\% CI: $2.3-138, p=0.0060)$, and 1.5 (95\% CI: $0.7-3.2, p=.3568)$, respectively. The difference from placebo in change from baseline in the double-blind period in HAM-A total score was -3.2 $(p<0.0001)$ in the working population overall, while the effect of vortioxetine versus placebo among professionals was $-5.6(p=0.0021)$ (Table 4$)$. The effect in the total population was $-3.2(p<0.0001)$. In terms of quality of life and global functioning, a significant effect versus placebo was also observed in working patients on the three SF-36 domains and the SDS, with a particularly pronounced effect in the professional subgroup. While the effect on SF-36 mental health index, SF-36 role-emotional, and SF-36 social functioning in the total population was 2.7 $(p=0.0058), 2.6 \quad(p=0.0051)$, and $2.8 \quad(p=0.0009)$, respectively, the effect among professionals was 8.2 $(p=0.0004), 6.2 \quad(p=0.0029)$, and $4.3 \quad(p=0.0266)$, 

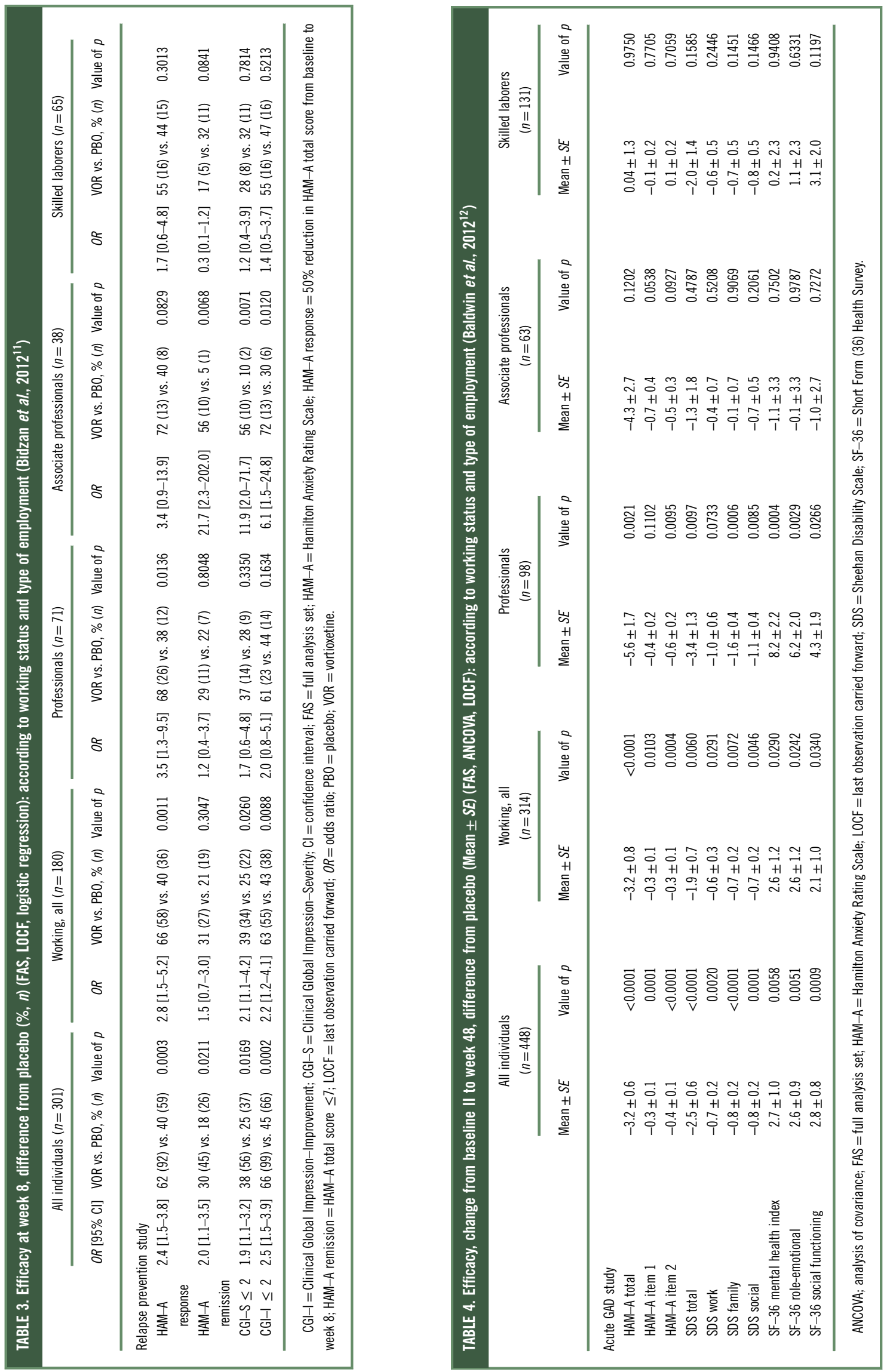
respectively. For SDS total score, SDS work, SDS social, and SDS family, the effect in the total population was $\quad-2.5 \quad(p<0.0001), \quad-0.7 \quad(p=0.0020), \quad-0.8$ $(p<0.0001)$, and $-0.8(p=0.0001)$, respectively, while the effect among professionals was $-3.4(p=0.0097),-1.0$ $(p=0.0733),-1.6(p=0.0006)$, and $-1.1 \quad(p=0.0085)$, respectively.

\section{Discussion}

To our knowledge, this is the first time an analysis has been conducted that sought to determine the effect of working status or educational enrollment on health outcomes with a pharmacological intervention in adults with GAD. The large sample size (i.e., approximately 1,000 subjects) provided a unique opportunity to evaluate the efficacy of vortioxetine in working patients with GAD. In accordance with our hypothesis, we observed a greater effect in working GAD patients not only on anxiety symptoms, but also in terms of global functioning and the quality of life experienced. This more pronounced multidomain effect was observed not only in an acute GAD population after 8 weeks of treatment with vortioxetine but also in a remitted GAD population treated with vortioxetine up to 48 weeks after remission. The more pronounced effect on anxiety symptoms in the working population also resulted in a greater relapse prevention effect when treated with vortioxetine. Consistent across endpoints in both studies, a greater effect was observed among working GAD patients in either professional or associate professional positions (or both) compared to the total GAD population. This foregoing finding is consistent with our previous research in working MDD patients where a much greater antidepressant and procognitive effect of vortioxetine was also observed among patients in professional positions. ${ }^{14}$

The DCS model brings into play such work characteristics as job demand, decision authority, skill discretion, and social support from colleagues to explain health outcomes and work productivity and serves as an explanatory conceptual framework. Specifically, the Whitehall II study identified a lack of social support from managers and colleagues as a risk factor for development of psychiatric disorders after adjustment for baseline disease severity. ${ }^{16}$ While our studies did not collect data on measures identical to those in the Whitehall II study, it could be speculated that subjects in professional and associate professional positions benefit from an optimal balance of these intersecting and dynamic factors. For example, the construct of resiliency, which overlaps with measurable dimensions of personality, has been shown to be associated with greater levels of seniority in preliminary studies. ${ }^{17}$ Indeed, the notion of locus of control as a resiliency and/or vulnerability factor would imply that individuals in more leadership-related positions are more likely, when compared to individuals in lower organizational structure positions or not in education, to be susceptible to changes and vicissitudes as well as changes to the organization both intrinsic and extrinsic. As a consequence, interventional outcomes, pharmacologic or nonpharmacologic, may be more effective in mitigating anxiety in individuals with a higher internal locus of control. A related issue is that the domain of anxiety is thought to be a result of real and/or perceived threat. According to the Research Domain Criteria (RDoC), GAD is a disturbance within the neurobiology of threat. Individuals who are higher in the organizational structure, because of perceived or real opportunity, may be more likely to benefit from threat-reduction interventions.

The mechanism mediating vortioxetine's anxiolytic effects in adults with GAD and MDD are unknown. Notwithstanding, the working hypothesis has been that the anxiolytic effects are due to a combination of effects at the 5 -HT reuptake transporter, as well as the $5-\mathrm{HT}_{1 \mathrm{~A}}$ agonist. ${ }^{18}$ Non-mutually-exclusive mechanisms that may also be pertinent are the indirect effects of vortioxetine on glutamatergic signaling via interneuron $5-\mathrm{HT}_{3}$ antagonism. ${ }^{18}$ It is posited that the glutamatergic effects facilitate neuroplastic changes in brain regions relevant to maladaptive anxious states (e.g., amygdala). ${ }^{19}$ It is further conjectured, informed by results from preclinical and clinical studies, that vortioxetine's beneficial effects across subjective and objective measures of cognitive functioning may also contribute to anxiolysis. For example, cognitive appraisal and disturbance in executive function (e.g., ruminations) are paradigmatic disturbances in anxiety disorders. ${ }^{20}$ The foregoing cognitive functions are possibly benefited by vortioxetine, resulting in reduced subjective distress. ${ }^{21}$ It is further hypothesized that combining behavioral activation (e.g., via increased engagement with workplace and/or educational pursuits) may facilitate the foregoing neurobiological and procognitive effects observed with vortioxetine.

We have previously proposed that ongoing participation in the workforce can be conceptualized as a form of behavioral activation and that this could explain the remarkable differences observed in the effect of vortioxetine on depressive symptoms and cognitive functioning in working MDD patients. ${ }^{14}$ Given the substantial overlap between GAD and MDD, it is reasonable to assume that such additive benefits also extend to working patients with $\mathrm{GAD}$; in other words, a synergistic effect may be at play where greater support and reward at work interacts with the anxiolytic effect of vortioxetine. Finally, while the present GAD studies did not specifically assess the effect on cognitive functioning, the effect of vortioxetine on cognitive functioning in MDD is well-established and may also play a role for working GAD patients. ${ }^{22,23}$ 
Our findings could have important implications from the societal and cost perspectives. The humanistic and economic burden of GAD is well-characterized. ${ }^{24,25}$ The human burden not only relates to the aforementioned impairments in role functioning but also importantly reduced health-related quality of life. Studies using the same measure of quality of life (i.e., SF-36) as in our studies have identified impairments in all dimensions of quality of life (general health perception, physical functioning, role-physical, bodily pain, mental health, social functioning, role-emotional, and vitality) in patients with pure GAD and greater impairment than in patients with pure MDD. ${ }^{4}$ The direct utilization of healthcare resources is another important component of the total expenditures in addition to the impact of anxiety on work productivity. ${ }^{24}$ For example, a US-based study reported incremental healthcare costs of $\$ 2,138$ for patients with GAD, while a study in Germany reported a twofold higher than average number of visits to primary care practice. ${ }^{26,27}$ Targeted interventions, pharmacological and/or nonpharmacological, that effectively address not only anxiety symptoms but also psychosocial impairment in GAD are likely to be both cost-effective and to improve patient-reported outcomes.

\section{Limitations}

There are some limitations to this research that would affect the inference and interpretation of the data. All analyses have been conducted post hoc and as such prevent any conclusive statements about the unique effect of vortioxetine in the working GAD population. Rather, the analyses should be considered hypothesis-generating for further research. Additionally, the number of subjects in the associate professional employment group was relatively small, which may have affected the statistical significance given the large effect sizes observed for most endpoints. A direct statistical comparison between working and nonworking GAD patients could also be of interest; however, this study was not designed to address this comparison. Furthermore, working GAD patients are likely to differ from nonworking patients not only by their work status but also by their educational achievement, family and friends support, personal motivation, and a number of other personal characteristics that can influence employment. Some of these personal characteristics may be very difficult to measure and thereby capture in a clinical study. Finally, our research is limited by the general limitations of randomized clinical trials in GAD.

\section{Conclusions}

In conclusion, this is the first report to evaluate the effect of a pharmacological intervention on measures of anxiety, quality of life, and functioning in adults with
$\mathrm{GAD}$ as a function of working status. When compared to previous reports of the effect of vortioxetine in patients with GAD (working and nonworking), a more pronounced effect was observed among working individuals treated with vortioxetine compared to the total population. Given the substantial humanistic and economic costs of GAD, our findings could be relevant for further research into the clinical value and costeffectiveness of targeted combined interventions, as well as pharmacological and psychosocial support in the workplace.

\section{Disclosures}

Ioana Florea, Henrik Loft, and Michael Cronquist Christensen are employees of $\mathrm{H}$. Lundbeck A/S. Dr. McIntyre reports grants and personal fees from H. Lundbeck A/S, grants and personal fees from Pfizer, grants and personal fees from Takeda, grants and personal fees from Eli Lilly, grants and personal fees from Otsuka, grants and personal fees from Bristol-Myers Squibb, grants and personal fees from Janssen, grants and personal fees from Purdue, and grants and personal fees from Allergan, outside the submitted work.

Data for this study were from clinical studies sponsored by H. Lundbeck A/S, Valby, Denmark, and Takeda Pharmaceuticals Inc., Deerfield, Illinois. M.C. Christensen, H. Loft, and I. Florea are employees of H. Lundbeck A/S.

ClinicalTrials.gov identifiers: no. NCT00744627 (study 311), no NCT00788034 (study 12473A).

\section{REFERENCES:}

1. Kessler RC, DuPont RL, Berglund P, Wittchen HU. Impairment in pure and comorbid generalized anxiety disorder and major depression at 12 months in two national surveys. Am J Psychiatry. 1999; 156(12): 1915-1923.

2. Kessler RC, Chiu WT, Demler O, Merikangas KR, Walters EE. Prevalence, severity, and comorbidity of 12-month DSM-IV disorders in the National Comorbidity Survey Replication. Arch Gen Psychiatry. 2005; 62(6): 617-627.

3. Carter RM, Wittchen HU, Pfister H, Kessler RC. One-year prevalence of subthreshold and threshold DSM-IV generalized anxiety disorder in a nationally representative sample. Depress Anxiety. 2001; 13(2): 78-88.

4. Wittchen HU, Carter RM, Pfister H, Montgomery SA, Kessler RC. Disabilities and quality of life in pure and comorbid generalized anxiety disorder and major depression in a national survey. Int Clin Psychopharmacol. 2000; 15(6): 319-328.

5. Karasek R, Theorell T 1990 Healthy Work: Stress Productivity and the Reconstruction of Working Life. New York: Basic Books.

6. Siegrist J. Adverse health effects of high-effort/low-reward conditions. J Occup Health Psychol. 1996; 1: 27-41.

7. Griffin JM, Greiner BA, Stansfeld SA, Marmot M. The effect of self-reported and observed job conditions on depression and anxiety symptoms: a comparison of theoretical models. J Occup Health Psychol. 2007; 12(4): 334-349. 
8. Karasek R. Job demands, job decision latitude, and mental strain: Implications for job redesign. Adm Sci Q. 1979; 24: 285-307.

9. Stansfeld SA, North FM, White I, Marmot MG. Work characteristics and psychiatric disorder in civil servants in London. J Epidemiol Community Health. 1995; 49(1): 48-53.

10. Ferrie JE, Shipley MJ, Stansfeld SA, Marmot MG. Effects of chronic job insecurity and change in job security on self reported health, minor psychiatric morbidity, physiological measures, and health related behaviours in British civil servants: the Whitehall II study. J Epidemiol Community Health. 2002; 56(6): 450-454.

11. Bidzan L1, Mahableshwarkar AR, Jacobsen P, Yan M, Sheehan DV. Vortioxetine (Lu AA21004) in generalized anxiety disorder: results of an 8-week, multinational, randomized, double-blind, placebocontrolled clinical trial. Eur Neuropsychopharmacol. 2012; 22(12): 847-857.

12. Baldwin DS, Loft H, Florea I. Lu AA 21004, a multimodal psychotropic agent, in the prevention of relapse in adult patients with generalized anxiety disorder. Int Clin Psychopharmacol. 2012 27(4): 197-207.

13. Pae CU, Wang SM, Han C, et al. Vortioxetine, a multimodal antidepressant for generalized anxiety disorder: a systematic review and meta-analysis. J Psychiatr Res. 2015; 64: 88-98.

14. McIntyre RS, Florea I, Tonnoir B, Loft H, Lam RW, Christensen MC. Efficacy of vortioxetine on cognitive functioning in working patients with major depressive disorder. J Clin Psychiatry. 2016; 78(1): 115-121

15. Wade AG, Fernández JL, François C, Hansen K, Danchenko N, Despiegel N. Escitalopram and duloxetine in major depressive disorder: a pharmacoeconomic comparison using UK cost data. Pharmacoeconomics. 2008; 26(11): 969-981.

16. Stansfeld SA, Fuhrer R, Shipley MJ, Marmot MG. Work characteristics predict psychiatric disorder: prospective results from the Whitehall II Study. J Occup Env Med. 1999; 56(5): 302-307.

17. Hsieh HF, Chang SC, Wang HH. The relationships among personality, social support, and resilience of abused nurses at emergency rooms and psychiatric wards in Taiwan. Women Health. 2017; 57(1): 40-51
18. Sanchez C, Asin KE, Artigas F. Vortioxetine, a novel antidepressant with multimodal activity: review of preclinical and clinical data. Pharmacol Ther. 2015; 145: 43-57.

19. van Eijndhoven P, van Wingen G, Fernández G, et al. Amygdala responsivity related to memory of emotionally neutral stimuli constitutes a trait factor for depression. NeuroImage. $2011 ; \mathbf{5 4}(2)$ : 1677-1684.

20. Vălenaş SP, Szentágotai-Tătar A, Grafton B, Notebaert L, Miu AC, MacLeod C. Prediction of pre-exam state anxiety from ruminative disposition: the mediating role of impaired attentional disengagement from negative information. Behav Res Ther. 2017; 91: $102-110$

21. McIntyre RS, Xiao HX, Syeda K, et al. The prevalence, measurement, and treatment of the cognitive dimension/domain in major depressive disorder. CNS Drugs. 2015; 29(7): 577-589.

22. McIntyre RS, Lophaven S, Olsen CK. A randomized, double-blind, placebo-controlled study of vortioxetine on cognitive function in depressed adults. Int J Neuropsychopharmacol. 2014; 17(10): 1557-1567.

23. Mahableshwarkar AR, Zajecka J, Jacobson W, Chen Y, Keefe RS. A randomized, placebo-controlled, active-reference, double-blind, flexible-dose study of the efficacy of vortioxetine on cognitive function in major depressive disorder. Neuropsychopharmacology. 2015; 22(8): 2025-2037.

24. Revicki DA, Travers K, Wyrwich KW, et al. Humanistic and economic burden of generalized anxiety disorder in North America and Europe. J Affect Disord. 2012; 140(2): 103-112.

25. Hoffman DL, Dukes EM, Wittchen HU. Human and economic burden of generalized anxiety disorder. Depress Anxiety. 2008 25(1): 72-90.

26. Marciniak MD, Lage MJ, Dunayevich E, et al. The cost of treating anxiety: the medical and demographic correlates that impact total medical costs. Depress Anxiety. 2005; 21(4): 178-184.

27. Wittchen HU, Kessler RC, Beesdo K, Krause P, Höfler M, Hoyer J. Generalized anxiety and depression in primary care: prevalence, recognition, and management. JClin Psychiatry. 2002; 63(Suppl 8): 24-34. 